\title{
Low muscle mass and body composition analysis in a group of postmenopausal women affected by primary Sjögren's syndrome
}

\author{
Suhel Gabriele Al Khayyat ID , Paolo Falsetti ID , Edoardo Conticini ID, Roberto D'Alessandro ID, \\ Francesca Bellisai ${ }^{\mathrm{D}}$, Stefano Gentileschi ${ }^{\mathrm{ID}}$, Caterina Baldi ID, Marco Bardelli ID, \\ Luca Cantarini ID , Bruno Frediani ID
}

Department of Medicine, Surgery and Neurosciences, Rheumatology Unit, University of Siena, Italy

\begin{abstract}
Objectives: Sarcopenia is the pathological reduction of skeletal muscle mass and strength. This condition is often underestimated in clinical practice, particularly in connective tissue diseases. The purpose of this study is to evaluate the prevalence of low muscle mass in primary Sjögren's syndrome (pSS) and to explore the relationships linking muscles and bone tissue.

Material and methods: Twenty-eight postmenopausal pSS patients were matched with 30 healthy controls and their body composition analysis was performed by dual-energy X-ray absorptiometry to investigate for sarcopenia considering appendicular lean mass (ALM) and the skeletal muscle mass index (SMI) as references. Bone mineral density analysis of lumbar spine (L1-L4), whole femur, femoral neck and whole body was also performed. Linear regression was used to assess the relationship between body composition and bone mineralization.

Results: Low muscle mass was significantly higher in the pSS group compared to controls whether expressed as ALM, SMI [odds ratio $(\mathrm{OR})=18.40$, confidence interval $(\mathrm{CI}): 4.84-72.08, p<0.0001$ ] or considering total body lean masses. Lean masses appeared to be the best estimators of bone mineralization: total lean body mass (TLBM) lumbar spine $R^{2}=0.72, p<0.0001$; TLBM femoral neck $R^{2}=0.36, p<0.004$; lean mass of upper limbs lumbar spine $R^{2}=0.70, p<0.0001$; femoral neck $R^{2}=0.66$; lean mass of lower limbs lumbar spine $R^{2}=0.66, p<0.0001$; femoral neck $R^{2}=0.44$, $p=0.008)$. Primary Sjögren's syndrome patients had a significantly higher android/gynoid fat ratio compared to controls.

Conclusions: Female pSS patients have lower muscle mass compared to healthy controls and are exposed to a higher risk of developing sarcopenia than healthy subjects. Our research demonstrates that the amount of lean tissue is the main predictor of bone mineralization in pSS.

Key words: bone mineral density, osteoporosis, sarcopenia, primary Sjögren's syndrome.
\end{abstract}

\section{Introduction}

Sarcopenia is a clinical condition defined by the European Consensus Group [1] as the reduction of skeletal muscle mass, associated with poor physical performance measured as low grip strength and gait speed. Low muscle mass represents a transversal problem in medicine, mainly affecting geriatric patients simultaneously suffering from many disorders such as chronic obstructive pul- monary disease (COPD), chronic heart failure and neurodegenerative diseases, defining the classic phenotype of the complex patient.

In recent decades, scientific attention has gradually increased towards sarcopenia in relation to several rheumatological conditions: the most robust evidence has been reported for rheumatoid arthritis (RA) [2] and systemic sclerosis [3], while contraddictory evidences have been reported for spondyloarthropaties (SA) [4]. 
A high inflammatory load, partly supported by the rheumatological diseases themselves and assisted by an obesity-prone body composition, has been linked to higher prevalence of skeletal muscle loss [5].

No data exist about the prevalence of sarcopenia in patients affected by primary Sjögren's syndrome (pSS): in this regard, we aimed to assess whether patients suffering from this condition may display a reduced muscle mass and whether any correlation exists between body composition and bone mineral density.

\section{Material and methods}

\section{Study population}

The authors retrospectively evaluated body composition and bone mineral density (BMD) of 28 postmenopausal women diagnosed in our department with pSS according to American College of Rheumatology/European League against Rheumatisms (ACR/EULAR) 2016 classification criteria [6]

The authors considered only women whose densitometry analysis was performed within 6.5 months (SD \pm 9.1 ) from pSS diagnosis. The exclusion criterion was the presence of any demineralizing condition, such as heparin therapy, corticosteroid use, COPD, chronic heart failure, malignancies, or cachexia.

\section{Control group}

Thirty postmenopausal women who had been evaluated with dual-energy X-ray absorptiometry (DXA) in our department for age-related issues, matched for age and gender, were randomly chosen from our records. None of them reported a major condition unbalancing bone homeostasis.

\section{Methods}

Detailed medical history, weight, height, and BMI were collected from patients and their sera were tested for autoimmunity with standard techniques: anti-nucleus antibodies (ANA) were detected by indirect immunofluorescence on HEp-2 cells (Euroimmun, Lübeck, Germany), anti-extractable nuclear antigens (ENA) using fluorescent enzyme immunoassay (FEIA - Thermo Fisher).

Minor salivary glands biopsy was performed according to Guevara-Gutiérrez technique [7] and samples were evaluated using the focus score (FS) and the Chisholm and Mason (CM) grading system. Samples were considered positive for pSS when $F S \geq 1$ and $C M \geq 3$. Bone mineral density and body composition were assessed with DXA using a Lunar Expert version $1.72{ }^{\circledR}$.
According to the manufacturer, before each examination routine quality control (QC) was performed. The following regions were evaluated:

- whole body,

- whole left femur,

- neck left femur,

- lumbar spine (L1-L4).

Bone mineral density was expressed in $\mathrm{g} / \mathrm{cm}^{2}$. A diagnosis of osteoporosis was made when the $t$-score of patients deviated below -2.5 standard deviations (SD) compared with the reference population according to the World Health Organization (WHO) criteria.

Body composition of pSS patients and controls was compared by fat and lean masses of total body, upper and lower limb regions. Appendicular lean mass (ALM, $\mathrm{kg} / \mathrm{m}^{2}$ ) was calculated as the sum of fat-free mass minus bone mineral content of lower and upper limbs, skeletal muscle mass index (SMI, $\mathrm{kg} / \mathrm{m}^{2}$ ) was calculated as ALM divided by height squared according to Baumgartner's criteria [8], fat mass index FMI $\left(\mathrm{kg} / \mathrm{m}^{2}\right)$ as total fat mass divided by height squared, and fat-free mass index (FFMI kg/m²) as fat-free tissue divided by height squared. A low muscle mass was identified for women when it was found to be 2 SD below the mean of young adults (women: $<5.5 \mathrm{~kg} / \mathrm{m}^{2}$ ) [8].

This study was conducted retrospectively and in accordance with the Declaration of Helsinki, all patients provided written consent to undergo clinical routine examination.

\section{Statistical analysis}

Statistical analysis was performed using GraphPad Prism version 8.4.2 for Windows, GraphPad Software, San Diego, California USA, www.graphpad.com. Mean total lean mass $(\mathrm{kg}), \mathrm{ALM}\left(\mathrm{kg} / \mathrm{m}^{2}\right)$, skeletal muscle mass index (SMI, $\left.\mathrm{kg} / \mathrm{m}^{2}\right)$, fat-free tissue ( $\left.\mathrm{kg}\right)$, fat-free mass index (FFMI, $\left.\mathrm{kg} / \mathrm{m}^{2}\right)$, total fat mass $(\mathrm{kg})$, body fat percentage (BF, \%), fat mass index (FMl, $\left.\mathrm{kg} / \mathrm{m}^{2}\right)$, and total skeletal muscle mass $(\mathrm{kg})$, calculated according to Kim et al. [9] were compared between pSS and controls using the t-test for parametric data or the Mann-Whitney $U$ test.

Subsequently, Fisher's exact test was carried out to establish sarcopenia's prevalence in the studied population. Pearson's and Spearman's tests were used to evidence any correlation between the considered variables of body composition and BMD of the studied regions.

A bivariate regression analysis was conducted using BMD of the lumbar spine and femoral neck as the dependent variables, while total skeletal muscle mass, total lean mass, SMI, total fat mass, total body fat percentage, upper lean mass, lower limbs lean mass, upper limbs fat mass, lower limbs fat mass, upper limbs fat free mass, lower limbs fat free mass, android fat percentage, gynoid 
fat percentage, $\mathrm{A} / \mathrm{G}$ ratio, $\mathrm{BMI}$, weight, $\mathrm{FFMI}$ and $\mathrm{FMI}$ were identified as the independent variables.

A multivariate regression analysis was performed to identify the best estimators of BMD considering age as an exogenous variable and introducing it in a stepwise manner. Lastly, we checked which model acted as the best predictor using the extra sum-of-squares -test.

\section{Results}

Epidemiological features and body composition characteristics of the studied populations are summarized in Table I.

Statistically significant differences were found between pSS and the control group for ALM, SMI, total skeletal muscle mass and android/gynoid fat distribution. Appendicular lean mass and SMI were significantly lower in pSS compared to the control group, as well as total skeletal muscle mass.

No difference in postmenopausal age was evidenced between cases and controls. Conversely, A/G ratio was identified as significantly higher in our patients.

The Mann-Whitney $U$ test or Student's t-test was used to compare appendicular lean mass, SMI and total skeletal muscle mass, evidencing a statistically significant lower muscle mass in pSS patients compared to the control group. Subsequently Fisher's exact test evidenced that $80 \%$ of pSS patients had a low muscle mass expressed as $\mathrm{SMI}<5.5 \mathrm{~kg} / \mathrm{m}^{2}$ compared to healthy individuals (20\% of low muscle mass) [odds ratio $(\mathrm{OR})=$ 18.40 confidence interval $(\mathrm{Cl}): 4.84-72.08, p<0.0001]$.

Significant correlations between body composition variables and bone mineral density were found in pSS patients: higher correlations were identified for lean masses considered as total body (TLBM), lean mass of upper limbs (LMUL) and lean mass of lower limbs (LMLL).

Fat-free masses, which are the lean masses plus bone mineral content, displayed a high correlation with bone mineral densities of nearly every studied region. Appendicular lean mass and SMI showed a correlation only for femoral BMDs. Weight and BMI were highly correlated with bone mineralization, although the latter only with femoral BMDs. Fat masses did not display any correlation with BMD. A/G ratio correlated with lumbar spine and whole-body BMDs (Table II).

From the bivariate regression analysis, we concluded that lean masses had higher goodness of fit as bone mineral density predictors; although other variables, particularly weight and BMI, acted in a statistically significant fashion as feasible estimators, their $R^{2}$ did not reach significant values. Appendicular lean mass and SMI acted as poor predictors of femoral BMD and they
Table I. Epidemiological and body composition features of primary Sjögren's syndrome patients and controls

\begin{tabular}{|c|c|c|}
\hline Parameter & pSS patients & Controls \\
\hline Number & 28 & 30 \\
\hline Gender & $\mathrm{F}$ & $\mathrm{F}$ \\
\hline Age & $64.31 \pm 9.7$ & $59.90 \pm 3.8$ \\
\hline Weight [kg] & $65.81 \pm 12.6$ & $67.38 \pm 16.9$ \\
\hline Height [cm] & $162.5 \pm 7.43$ & $162.3 \pm 6.03$ \\
\hline $\begin{array}{l}\text { Body mass index } \\
{\left[\mathrm{BMl}, \mathrm{kg} / \mathrm{m}^{2}\right]}\end{array}$ & $24.86 \pm 4.1$ & $25.03 \pm 6.1$ \\
\hline Postmenopausal age & $51.09 \pm 3.19$ & $50.22 \pm 3.04$ \\
\hline \multicolumn{3}{|l|}{ Body composition analysis } \\
\hline $\begin{array}{l}\text { Total lean body mass } \\
{[\text { TLBM, kg] }}\end{array}$ & $38.88 \pm 6.73$ & $39.10 \pm 4.83$ \\
\hline Body fat [BF, \%] & $36.91 \pm 8.68$ & $39.35 \pm 8.99$ \\
\hline $\begin{array}{l}\text { Appendicular lean mass } \\
{\left[\mathrm{ALM}, \mathrm{kg} / \mathrm{m}^{2}\right]}\end{array}$ & $11.87 \pm 3.42^{\star \star}$ & $16.84 \pm 2.96^{* *}$ \\
\hline $\begin{array}{l}\text { Skeletal muscle mass } \\
\text { index }\left[\mathrm{SMI}, \mathrm{kg} / \mathrm{m}^{2}\right]\end{array}$ & $4.47 \pm 1.18^{\star \star}$ & $6.38 \pm 0.96^{\star *}$ \\
\hline Fat-free tissue [kg] & $39.92 \pm 6.66$ & $42.17 \pm 5.51$ \\
\hline $\begin{array}{l}\text { Fat-free mass index } \\
{\left[\mathrm{FFMl}, \mathrm{kg} / \mathrm{m}^{2}\right]}\end{array}$ & $14.58 \pm 3.33$ & $15.65 \pm 1.60$ \\
\hline Total fat mass [kg] & $23.99 \pm 9.82$ & $26.98 \pm 13.12$ \\
\hline Body fat percentage [BF, \%] & $36.91 \pm 8.68$ & $39.35 \pm 8.99$ \\
\hline $\begin{array}{l}\text { Fat mass index } \\
{\left[\mathrm{FMl}, \mathrm{kg} / \mathrm{m}^{2}\right]}\end{array}$ & $9.07 \pm 3.53$ & $10.31 \pm 4.81$ \\
\hline $\begin{array}{l}\text { Total skeletal muscle } \\
\text { mass [kg] }\end{array}$ & $13.10 \pm 3.94^{* *}$ & $16.76 \pm 3.29^{\star *}$ \\
\hline Android fat [\%] & $4.61 \pm 11.58$ & $43.48 \pm 43.88$ \\
\hline Gynoid fat [\%] & $41.91 \pm 7.25$ & $43.91 \pm 7.31$ \\
\hline $\begin{array}{l}\text { Android/gynoid fat ratio } \\
{[\text { A/G ratio }]}\end{array}$ & $1.8 \pm 0.47^{\star *}$ & $1 \pm 0.39^{\star *}$ \\
\hline
\end{tabular}

did not reach statistical significance as lumbar spine BMD estimators (Table III).

Subsequently, a multivariate model including age as an independent variable was built and a comparison between the two regression models was made using the extra sum-of-squares $f$-test, concluding that the multivariate model including age acted as the best BMD predictor for every studied region ( $p<0.05$; the null hypothesis where $\mathrm{HO}=$ the simpler model is correct was rejected).

Age always acted as negatively related to bone mineral densities. Absolute $t$-values for lean masses of body composition were higher than those of the age variable, suggesting a greater impact of lean masses in BMD prediction compared to age (Table IV). 
Table II. Body mass composition correlations with bone mineral density of studied regions among our cohort of primary Sjögren's syndrome patients

\begin{tabular}{|c|c|c|c|c|}
\hline Parameter & Whole femur BMD & Femoral neck BMD & Lumbar spine BMD & Whole body BMD \\
\hline Whole body BF [\%] & 0.29 & 0.09 & 0.18 & 0.10 \\
\hline Total mass whole body $[\mathrm{kg}]$ & $0.48^{\star}$ & $0.54^{\star *}$ & $0.56^{\star *}$ & $0.62^{\star *}$ \\
\hline Fat [kg] & 0.32 & 0.24 & 0.42 & 0.32 \\
\hline Lean total body [kg] & $0.51^{\star \star}$ & $0.46^{*}$ & $0.64^{\star *}$ & $0.51^{\star *}$ \\
\hline Fat free tissue total body [kg] & $0.42^{*}$ & $0.46^{*}$ & $0.63^{* *}$ & $0.63^{\star *}$ \\
\hline Android BF [\%] & $0.50^{*}$ & 0.36 & 0.35 & 0.22 \\
\hline Gynoid BF [\%] & 0 & -0.13 & -0.17 & -0.12 \\
\hline Torso BF [\%] & $0.48^{*}$ & $0.45^{*}$ & 0.26 & 0.21 \\
\hline Total mass torso [kg] & 0.21 & 0.33 & 0.38 & $0.49^{\star *}$ \\
\hline Fat torso $[\mathrm{kg}]$ & 0.37 & 0.32 & 0.37 & $0.42^{*}$ \\
\hline Lean torso [kg] & 0.14 & 0.35 & $0.53^{*}$ & $0.50^{\star *}$ \\
\hline Fat free tissue torso [kg] & 0.16 & 0.26 & $0.59^{\star *}$ & $0.42^{*}$ \\
\hline Lower limbs total mass [kg] & 0.37 & $0.55^{* *}$ & 0.36 & $0.46^{*}$ \\
\hline Lower limbs BF [\%] & 0.28 & 0.14 & 0.02 & -0.12 \\
\hline Fat lower limbs [kg] & 0.18 & 0.06 & 0.15 & 0.13 \\
\hline Lean lower limbs [kg] & 0.38 & $0.52^{* *}$ & $0.58^{\star \star}$ & 0.58 \\
\hline Fat free tissue lower limbs [kg] & 0.39 & $0.53^{* *}$ & $0.59^{\star *}$ & $0.60^{\star *}$ \\
\hline Upper limbs BF [\%] & 0.06 & -0.11 & 0.09 & 0.01 \\
\hline Upper limbs total mass [kg] & $0.43^{*}$ & $0.50^{* *}$ & 0.26 & 0.03 \\
\hline Fat upper limbs [kg] & 0.32 & 0.14 & 0.21 & 0.33 \\
\hline Lean upper limbs [kg] & $0.60^{\star \star}$ & $0.62^{* *}$ & $0.69^{\star *}$ & $0.59^{\star *}$ \\
\hline Fat-free tissue upper limbs [kg] & $0.63^{\star *}$ & $0.63^{* *}$ & $0.52^{*}$ & $0.74^{\star *}$ \\
\hline ALM $\left[\mathrm{kg} / \mathrm{m}^{2}\right]$ & $0.54^{\star \star}$ & $0.53^{\star *}$ & 0.25 & 0.39 \\
\hline $\mathrm{SMI}\left[\mathrm{kg} / \mathrm{m}^{2}\right]$ & $0.51^{\star \star}$ & $0.47^{\star}$ & 0.37 & 0.29 \\
\hline $\mathrm{FMI}\left[\mathrm{kg} / \mathrm{m}^{2}\right]$ & 0.28 & $0.47^{\star}$ & 0.35 & 0.22 \\
\hline $\mathrm{FFMI}\left[\mathrm{kg} / \mathrm{m}^{2}\right]$ & $0.50^{*}$ & $0.51^{\star *}$ & 0.22 & $0.46^{*}$ \\
\hline $\mathrm{A} / \mathrm{G}$ ratio & 0.19 & 0.33 & $0.60^{* *}$ & $0.55^{\star *}$ \\
\hline TSMM & $0.57^{\star}$ & $0.55^{\star *}$ & $0.46^{\star}$ & $0.40^{*}$ \\
\hline BMI $\left[\mathrm{kg} / \mathrm{m}^{2}\right]$ & $0.40^{\star}$ & $0.43^{*}$ & $0.41(p=0.06)$ & 0.32 \\
\hline Weight (kg) & $0.42^{*}$ & $0.48^{*}$ & $0.53^{*}$ & $0.48^{*}$ \\
\hline
\end{tabular}

A/G ratio - android/gynoid fat ratio, $A L M$ - appendicular lean mass, $B M D$ - bone mineral density, $B M I$ - body mass index, FFMI - fat-free mass index, FMI - fat mass index, SMI - skeletal muscle mass index, TSMM - total skeletal muscle mass; ${ }^{*} p<0.05,{ }^{* *} p<0.005$.

\section{Discussion}

Primary Sjögren's syndrome is a chronic inflammatory autoimmune connective tissue disease of unknown etiology which is characterized by CD4+ T-helper, B-cell, macrophage, and dendritic cell infiltration of target organs, in which the dysfunction of the immune and cytokine system plays a pivotal role in supporting disease activity.

Many rheumatological diseases characterized by a high inflammatory load have been linked to a reduc- tion of BMD and lean masses. To date, no study has investigated the association between low muscle mass and pSS.

In our patients we documented a higher frequency of low SMI. We could speculate that classical risk factors for sarcopenia such as reduced physical activity [10], hormonal imbalance, high disease burden, altered body composition, inflammation [11], low vitamin D levels [12] and insulin resistance [13] are involved in reducing lean mass in pSS patients, alongside an altered 
Table III. Results of multiple simple linear bivariate regression of body mass composition analysis conducted by DXA as feasible predictors of bone mineral density of lumbar spine and femoral neck

\begin{tabular}{|c|c|c|c|c|c|c|}
\hline X Independent variable & $\begin{array}{c}\text { Y } \\
\text { Dependent } \\
\text { variable }\end{array}$ & Equation & $p$-value & $r^{2}$ & $\begin{array}{c}\text { Std. err. } \\
\text { of the } \\
\text { Y-intercept }\end{array}$ & $\begin{array}{c}\text { Number } \\
\text { of observed } \\
\text { values }\end{array}$ \\
\hline Lean total body mass [kg] & $\begin{array}{l}\text { Lumbar spine } \\
\text { BMD }\left[\mathrm{g} / \mathrm{cm}^{2}\right]\end{array}$ & $\begin{array}{c}Y=0.03235^{*} X- \\
0.1980\end{array}$ & 0.0002 & 0.52 & 0.2644 & 28 \\
\hline Lean total body mass [kg] & $\begin{array}{l}\text { Femoral neck } \\
\text { BMD }\left[\mathrm{g} / \mathrm{cm}^{2}\right]\end{array}$ & $\begin{array}{c}Y=0.009076^{*} X+ \\
0.5052\end{array}$ & 0.035 & 0.17 & 0.1561 & 28 \\
\hline Total body fat [\%] & $\begin{array}{l}\text { Lumbar spine } \\
\text { BMD }\left[\mathrm{g} / \mathrm{cm}^{2}\right]\end{array}$ & $\begin{array}{c}Y=0.003605^{*} X+ \\
0.8602\end{array}$ & 0.4191 & 0.03 & 0.1680 & 28 \\
\hline Total body fat [\%] & $\begin{array}{l}\text { Femoral neck } \\
\text { BMD }\left[\mathrm{g} / \mathrm{cm}^{2}\right]\end{array}$ & $\begin{array}{c}Y=0.005983^{*} X+ \\
0.6373\end{array}$ & 0.0697 & 0.12 & 0.1197 & 28 \\
\hline Total body fat mass [kg] & $\begin{array}{l}\text { Lumbar spine } \\
\text { BMD }\left[\mathrm{g} / \mathrm{cm}^{2}\right]\end{array}$ & $\begin{array}{c}Y=0.007294^{*} X+ \\
0.8231\end{array}$ & $0.054 \mathrm{~ns}$ & 0.18 & 0.2644 & 28 \\
\hline Total body fat mass [kg] & $\begin{array}{l}\text { Femoral neck } \\
\text { BMD }\left[\mathrm{g} / \mathrm{cm}^{2}\right]\end{array}$ & $\begin{array}{c}Y=0.007947^{\star} X+ \\
0.6674\end{array}$ & 0.005 & 0.27 & 0.06704 & 28 \\
\hline $\mathrm{SMI}\left[\mathrm{kg} / \mathrm{m}^{2}\right]$ & $\begin{array}{l}\text { Lumbar spine } \\
\text { BMD }\left[\mathrm{g} / \mathrm{cm}^{2}\right]\end{array}$ & $\begin{array}{c}Y=0.04188^{*} X+ \\
0.8126\end{array}$ & $0.0932 \mathrm{~ns}$ & 0.14 & 0.1098 & 28 \\
\hline $\mathrm{SMI}\left[\mathrm{kg} / \mathrm{m}^{2}\right]$ & $\begin{array}{l}\text { Femoral neck } \\
\text { BMD }\left[\mathrm{g} / \mathrm{cm}^{2}\right]\end{array}$ & $\begin{array}{c}Y=0.04818^{*} X+ \\
0.6506\end{array}$ & 0.0131 & 0.22 & 0.08185 & 28 \\
\hline $\mathrm{ALM}\left[\mathrm{kg} / \mathrm{m}^{2}\right]$ & $\begin{array}{l}\text { Lumbar spine } \\
\text { BMD }\left[\mathrm{g} / \mathrm{cm}^{2}\right]\end{array}$ & $\begin{array}{c}Y=0.02117^{\star} X+ \\
0.7451\end{array}$ & $0.06 \mathrm{~ns}$ & 0.19 & 0.1278 & 28 \\
\hline $\mathrm{ALM}\left[\mathrm{kg} / \mathrm{m}^{2}\right]$ & $\begin{array}{l}\text { Femoral neck } \\
\text { BMD }\left[\mathrm{g} / \mathrm{cm}^{2}\right]\end{array}$ & $\begin{array}{c}Y=0.02643^{*} X+ \\
0.5443\end{array}$ & 0.001 & 0.36 & 0.08185 & 28 \\
\hline TSMM & $\begin{array}{l}\text { Lumbar spine } \\
\text { BMD }[\mathrm{g} / \mathrm{cm} 2]\end{array}$ & $\begin{array}{c}Y=0.01967^{*} X+ \\
0.7373\end{array}$ & 0.003 & 0.21 & 0.1246 & 28 \\
\hline TSMM & $\begin{array}{l}\text { Femoral neck } \\
\text { BMD }\left[\mathrm{g} / \mathrm{cm}^{2}\right]\end{array}$ & $\begin{array}{c}Y=0.02391^{\star} X+ \\
0.5451\end{array}$ & 0.0008 & 0.38 & 0.08458 & 28 \\
\hline $\mathrm{FMI}\left[\mathrm{kg} / \mathrm{m}^{2}\right]$ & $\begin{array}{l}\text { Lumbar spine } \\
\text { BMD }\left[\mathrm{g} / \mathrm{cm}^{2}\right]\end{array}$ & $\begin{array}{c}Y=0.01672^{\star} X+ \\
0.8453\end{array}$ & 0.1162 & 0.12 & 0.09854 & 28 \\
\hline $\mathrm{FMI}\left[\mathrm{kg} / \mathrm{m}^{2}\right]$ & $\begin{array}{l}\text { Femoral neck } \\
\text { BMD }\left[\mathrm{g} / \mathrm{cm}^{2}\right)\end{array}$ & $\begin{array}{c}Y=0.01966^{\star} X+ \\
0.6797\end{array}$ & 0.0125 & 0.22 & 0.07117 & 28 \\
\hline $\mathrm{FFMI}\left[\mathrm{kg} / \mathrm{m}^{2}\right]$ & $\begin{array}{l}\text { Lumbar spine } \\
\text { BMD }\left[\mathrm{g} / \mathrm{cm}^{2}\right]\end{array}$ & $\begin{array}{c}Y=0.04188^{\star} X+ \\
0.8126\end{array}$ & $0.0932 \mathrm{~ns}$ & 0.14 & 0.1098 & 28 \\
\hline $\mathrm{FFMI}\left[\mathrm{kg} / \mathrm{m}^{2}\right]$ & $\begin{array}{l}\text { Femoral neck } \\
\text { BMD }\left[\mathrm{g} / \mathrm{cm}^{2}\right]\end{array}$ & $\begin{array}{c}Y=0.04818^{\star} X+ \\
0.6506\end{array}$ & 0.0131 & 0.22 & 0.08185 & 28 \\
\hline Total body fat-free tissue [kg] & $\begin{array}{l}\text { Lumbar spine } \\
\text { BMD }\left[\mathrm{g} / \mathrm{cm}^{2}\right]\end{array}$ & $\begin{array}{c}Y=0.02817^{\star} X- \\
0.1073\end{array}$ & 0.001 & 0.44 & 0.2882 & 28 \\
\hline Total body fat-free tissue [kg] & $\begin{array}{l}\text { Femoral neck } \\
\text { BMD }\left[\mathrm{g} / \mathrm{cm}^{2}\right]\end{array}$ & $\begin{array}{c}Y=0.01131^{\star} X+ \\
0.3956\end{array}$ & 0.004 & 0.28 & 0.1471 & 28 \\
\hline Lean mass upper limbs [kg] & $\begin{array}{l}\text { Lumbar spine } \\
\text { BMD }\left[\mathrm{g} / \mathrm{cm}^{2}\right]\end{array}$ & $\begin{array}{c}Y=0.1506^{*} X+ \\
0.3733\end{array}$ & 0.0007 & 0.48 & 0.1557 & 28 \\
\hline Lean mass upper limbs (kg) & $\begin{array}{l}\text { Femoral neck } \\
\text { BMD }\left[\mathrm{g} / \mathrm{cm}^{2}\right]\end{array}$ & $\begin{array}{c}Y=0.1161^{\star} X+ \\
0.3592\end{array}$ & 0.0001 & 0.52 & 0.09894 & 28 \\
\hline Lean mass lower limbs [kg] & $\begin{array}{l}\text { Lumbar spine } \\
\text { BMD }\left[\mathrm{g} / \mathrm{cm}^{2}\right]\end{array}$ & $\begin{array}{c}Y=0.07724^{*} X+ \\
0.1056\end{array}$ & 0.0006 & 0.46 & 0.2188 & 28 \\
\hline Lean mass lower limbs [kg] & $\begin{array}{l}\text { Femoral neck } \\
\text { BMD }\left[\mathrm{g} / \mathrm{cm}^{2}\right]\end{array}$ & $\begin{array}{c}Y=0.03545^{*} X+ \\
0.4358\end{array}$ & 0.0020 & 0.32 & 0.1246 & 28 \\
\hline Fat free tissue upper limbs [kg] & $\begin{array}{l}\text { Lumbar spine } \\
\text { BMD }\left[\mathrm{g} / \mathrm{cm}^{2}\right]\end{array}$ & $\begin{aligned} Y= & 0.1487^{\star} X+ \\
& 0.3348\end{aligned}$ & 0.0002 & 0.51 & 0.1485 & 28 \\
\hline Fat free tissue upper limbs [kg] & $\begin{array}{l}\text { Femoral neck } \\
\text { BMD }\left[\mathrm{g} / \mathrm{cm}^{2}\right]\end{array}$ & $\begin{aligned} Y= & 0.1090^{*} X+ \\
& 0.3590\end{aligned}$ & $<0.0001$ & 0.52 & 0.09629 & 28 \\
\hline
\end{tabular}


Table III. Cont.

\begin{tabular}{|c|c|c|c|c|c|c|}
\hline $\mathrm{X}$ Independent variable & $\begin{array}{c}\text { Y } \\
\text { Dependent } \\
\text { variable }\end{array}$ & Equation & $p$-value & $r^{2}$ & $\begin{array}{l}\text { Std. err. } \\
\text { of the } \\
\text { Y-intercept }\end{array}$ & $\begin{array}{c}\text { Number } \\
\text { of observed } \\
\text { values }\end{array}$ \\
\hline Fat free tissue lower limbs [kg] & $\begin{array}{l}\text { Lumbar spine } \\
\text { BMD }\left[\mathrm{g} / \mathrm{cm}^{2}\right]\end{array}$ & $\begin{aligned} Y= & 0.07304^{*} X+ \\
& 0.09543\end{aligned}$ & 0.0004 & 0.49 & 0.2094 & 28 \\
\hline Fat free tissue lower limbs [kg] & $\begin{array}{l}\text { Femoral neck } \\
\text { BMD }\left[\mathrm{g} / \mathrm{cm}^{2}\right]\end{array}$ & $\begin{aligned} Y= & 0.03436^{*} \mathrm{X}+ \\
& 0.4204\end{aligned}$ & 0.0013 & 0.34 & 0.1227 & 28 \\
\hline Android fat [\%] & $\begin{array}{l}\text { Lumbar spine } \\
\text { BMD }\left[\mathrm{g} / \mathrm{cm}^{2}\right]\end{array}$ & $\begin{array}{c}Y=0.002914^{*} X+ \\
0.8788\end{array}$ & $0.3632 \mathrm{~ns}$ & 0.04 & 0.1309 & 28 \\
\hline Android fat [\%] & $\begin{array}{l}\text { Femoral neck } \\
\text { BMD }\left[\mathrm{g} / \mathrm{cm}^{2}\right]\end{array}$ & $\begin{array}{c}Y=0.005888^{*} X+ \\
0.6190\end{array}$ & 0.0145 & 0.21 & 0.09462 & 28 \\
\hline Gynoid fat [\%] & $\begin{array}{l}\text { Lumbar spine } \\
\text { BMD }\left[\mathrm{g} / \mathrm{cm}^{2}\right]\end{array}$ & $\begin{array}{c}Y=0.004454^{*} X+ \\
1.188\end{array}$ & 0.5433 ns & 0.01 & 0.3147 & 28 \\
\hline Gynoid fat [\%] & $\begin{array}{l}\text { Femoral neck } \\
\text { BMD }\left[\mathrm{g} / \mathrm{cm}^{2}\right]\end{array}$ & $\begin{array}{c}Y=0.0005586^{*} X+ \\
0.8815\end{array}$ & $0.8911 \mathrm{~ns}$ & 0.00 & 0.1718 & 28 \\
\hline $\mathrm{A} / \mathrm{G}$ ratio & $\begin{array}{l}\text { Lumbar spine } \\
\text { BMD }\left[\mathrm{g} / \mathrm{cm}^{2}\right]\end{array}$ & $\begin{aligned} Y= & 0.1838^{*} X+ \\
& 0.6658\end{aligned}$ & 0.0827 ns & 0.15 & 0.1836 & 28 \\
\hline $\mathrm{A} / \mathrm{G}$ ratio & $\begin{array}{l}\text { Femoral neck } \\
\text { BMD }\left[\mathrm{g} / \mathrm{cm}^{2}\right]\end{array}$ & $\begin{aligned} Y= & 0.08419^{*} X+ \\
& 0.7015\end{aligned}$ & 0.1694 ns & 0.07 & 0.1142 & 28 \\
\hline BMI $\left(\mathrm{kg} / \mathrm{m}^{2}\right)$ & $\begin{array}{l}\text { Lumbar spine } \\
\text { BMD }\left[\mathrm{g} / \mathrm{cm}^{2}\right]\end{array}$ & $\begin{aligned} Y= & 0.01844^{\star} X+ \\
& 0.5447\end{aligned}$ & 0.06 & 0.17 & 0.2287 & 28 \\
\hline $\mathrm{BMI}\left(\mathrm{kg} / \mathrm{m}^{2}\right)$ & $\begin{array}{l}\text { Femoral neck } \\
\text { BMD }\left[\mathrm{g} / \mathrm{cm}^{2}\right]\end{array}$ & $\begin{aligned} Y= & 0.01572^{*} X+ \\
& 0.4672\end{aligned}$ & 0.02 & 0.19 & 0.1634 & 28 \\
\hline Weight (kg) & $\begin{array}{l}\text { Lumbar spine } \\
\mathrm{BMD}\left[\mathrm{g} / \mathrm{cm}^{2}\right]\end{array}$ & $\begin{array}{c}Y=0.007824^{*} X+ \\
0.4931\end{array}$ & 0.01 & 0.28 & 0.1860 & 28 \\
\hline Weight (kg) & $\begin{array}{l}\text { Femoral neck } \\
\text { BMD }\left[\mathrm{g} / \mathrm{cm}^{2}\right]\end{array}$ & $\begin{array}{c}Y=0.005803^{*} X+ \\
0.5228\end{array}$ & 0.02 & 0.23 & 0.1384 & 28 \\
\hline
\end{tabular}

A/G ratio - android/gynoid fat ratio, $A L M$ - appendicular lean mass, $B M D$ - bone mineral density, BMI - body mass index, FFMI - fat-free mass index, FMI - fat mass index, SMI - skeletal muscle mass index, TSMM - total skeletal muscle mass; $n s$ - not significant, ${ }^{*} p<0.05$.

gut microbiota, an impaired nutritional status [14] and altered glandular functions [15], which may occur in Sjögren's disease.

Indeed, many of our patients displayed low vitamin D levels (data not shown), and this could partially explain the reduction of lean tissue in pSS. Phenotypical changes may reflect pathological states that act simultaneously on bone, muscle and fat tissue where the bone-muscle unit cross-talk is bidirectional and one tissue influences the other [16].

The fat mass acts as an endocrinological tissue that may influence BMD, muscles, and body composition by secreting adipokines: the most studied are leptin and adiponectin; the former is known as an appetite suppressor and, even if its role in bone is not thoroughly defined yet [17], it seems to enhance energy consumption in skeletal muscle [18]. The latter has been negatively correlated with BMD [17], while its deficiency was linked to lower insulin sensitivity of muscle tissue [19].

The results derived by body composition analysis evidenced that, as already reported in the literature [20], lean tissues may act as good predictors of BMD, while a similar association was not found for BMI and patients' weight.

Although lean masses carried higher absolute $t$-values, adding age as an independent variable significantly ameliorated our models' prediction capabilities, allowing us to hypothesize that muscle tissue have a greater influence on BMD than BMI or weight and to speculate that, independently from age, physical activity may act as the best non-pharmacological measure against muscle and bone loss, in accordance with what is reported [21].

Moreover, 6 out of our patients (21\%) could be classified in the osteosarcopenic-obesity phenotype, i.e. BF\% $>40 \%$ for women [22], BMD (expressed as t-score at any site) lower than -1 SD in accordance with the WHO definition and $\mathrm{SMI}<5.45 \mathrm{~kg} / \mathrm{m}^{2}$ [23].

Though an increase in fracture risk is described for pSS [24], lean mass tissues have not been considered in these studies, and as suggested by Yu et al. [25], combining the presence of sarcopenia with FRAX could improve fracture risk prediction. The weight of low muscle mass in rheumatological diseases as a predictor of fractures has yet to be fully investigated. 
Table IV. Results of the multivariate regression analysis showing the lean masses and age estimators of lumbar spine and femoral neck bone mineral density $\left(\mathrm{g} / \mathrm{cm}^{2}\right)$

\begin{tabular}{|c|c|c|c|c|c|c|c|c|c|c|c|c|}
\hline $\mathrm{X} 1$ & $\mathrm{X} 2$ & Y & $\beta 0$ & $\beta 1$ & $\beta 2$ & $p$-value $\mathrm{F}$ & $r^{2}$ & $\begin{array}{c}\text { Std. err. } \\
\beta 1\end{array}$ & $\begin{array}{c}\text { Std. err. } \\
\beta 2\end{array}$ & $\begin{array}{l}|t| \\
\beta 1\end{array}$ & $\begin{array}{l}|t| \\
\beta 2\end{array}$ & $n$ \\
\hline $\begin{array}{l}\text { TLBM } \\
{[\mathrm{kg}]}\end{array}$ & Age (y) & $\begin{array}{c}\text { Lumbar } \\
\text { spine BMD } \\
{\left[\mathrm{g} / \mathrm{cm}^{2}\right]}\end{array}$ & 0.344 & 0.033 & -0.0093 & $<0.0001$ & 0.72 & 0.005 & 0.002 & 4.5 & 2.0 & 28 \\
\hline $\begin{array}{l}\text { TLBM } \\
{[\mathrm{kg}]}\end{array}$ & Age (y) & $\begin{array}{c}\text { Femoral } \\
\text { neck BMD } \\
{\left[\mathrm{g} / \mathrm{cm}^{2}\right]}\end{array}$ & 0.807 & 0.012 & -0.007 & 0.0046 & 0.36 & 0.003 & 0.002 & 0.0 & 0.0 & 28 \\
\hline $\begin{array}{l}\text { LMUL } \\
{[\mathrm{kg}]}\end{array}$ & Age (y) & $\begin{array}{c}\text { Lumbar } \\
\text { spine BMD } \\
{\left[\mathrm{g} / \mathrm{cm}^{2}\right]}\end{array}$ & 0.948 & 0.153 & -0.0095 & $<0.0001$ & 0.70 & 0.028 & 0.002 & 4.5 & 2.0 & 28 \\
\hline $\begin{array}{l}\text { LMUL } \\
{[\mathrm{kg}]}\end{array}$ & Age (y) & $\begin{array}{c}\text { Femoral } \\
\text { neck BMD } \\
{\left[\mathrm{g} / \mathrm{cm}^{2}\right]}\end{array}$ & 0.690 & 0.126 & -0.0058 & $<0.0001$ & 0.66 & 0.019 & 0.001 & 5.1 & 1.2 & 28 \\
\hline $\begin{array}{l}\text { LMLL } \\
{[\mathrm{kg}]}\end{array}$ & Age (y) & $\begin{array}{c}\text { Lumbar } \\
\text { spine BMD } \\
{\left[\mathrm{g} / \mathrm{cm}^{2}\right]}\end{array}$ & 0.647 & 0.078 & -0.0090 & $<0.0001$ & 0.66 & 0.015 & 0.002 & 4.1 & 2.0 & 28 \\
\hline $\begin{array}{l}\text { LMLL } \\
{[\mathrm{kg}]}\end{array}$ & Age (y) & $\begin{array}{c}\text { Femoral } \\
\text { neck BMD } \\
{\left[\mathrm{g} / \mathrm{cm}^{2}\right]}\end{array}$ & 0.734 & 0.039 & -0.0054 & 0.0008 & 0.44 & 0.009 & 0.002 & 3.4 & 1.2 & 28 \\
\hline TSMM & Age (y) & $\begin{array}{c}\text { Lumbar } \\
\text { spine BMD } \\
\text { [g/cm²] }\end{array}$ & 1.324 & 0.021 & -0.0098 & 0.0076 & 0.00 & 0.007 & 0.003 & 2.2 & 0.0 & 28 \\
\hline TSMM & Age (y) & $\begin{array}{c}\text { Femoral } \\
\text { neck BMD } \\
{\left[\mathrm{g} / \mathrm{cm}^{2}\right]}\end{array}$ & 0.781 & 0.023 & -0.0036 (ns) & 0.0013 & 0.44 & 0.006 & 0.002 & 3.8 & 1.2 & 28 \\
\hline
\end{tabular}

$\beta O-Y$-intercept, $\beta 1 / \beta 2$ - estimates of independent variables, Std. err - standard error, LTBM - lean total body mass, LMUL - lean mass of upper limbs, LMLL - lean mass of lower limbs, TSMM - total skeletal muscle mass, ns - not significant, |t| - t absolute value of the univariate analysis used to weight the impact of each independent variable on the model.

Altered body composition in RA has been correlated with the increase in IL-6 levels [26], insulin resistance and metabolic syndrome [27], thus partially accounting for the increased cardiovascular risk of these patients. The degree of inflammation in chronic inflammatory arthritis was directly correlated in several studies with obesity [28] and, as well as in RA, with prolongation of the QTc interval leading to malignant ventricular arrhythmias such as torsade de pointes [29].

Our patients displayed a statistically significantly higher $\mathrm{A} / \mathrm{G}$ ratio compared to controls, and android fat distribution is positively correlated with a higher risk of coronary disease in the literature [30].

Except for the study of Gravani et al. [31], which reported myo-intimal thickening of the arteries of patients with pSS, no other papers correlated an altered body composition and an altered cytokine pool with an increased risk of cardiovascular events in pSS patients.

\section{Conclusions}

At the present time, muscle, fat and skeletal tissue should be no longer considered as entities functional- ly separated from each other but rather as a syncytium with endocrine and immunological function, given the close interconnection of these systems.

This is the first study to document a high prevalence of reduced skeletal muscle mass in postmenopausal women recently diagnosed with pSS. Such patients appear to be at least as much at risk of sarcopenic syndrome as those with RA and systemic sclerosis, albeit perhaps due to different pathophysiological mechanisms.

This paper has several limitations: the considered cohort is too small to allow generalization of the results to every postmenopausal woman affected by pSs; moreover, due to its retrospective design, it was not possible to stratify patients according to organ involvement and disease activity. Therefore, prospective studies involving larger cohorts are needed to obtain higher quality of evidence.

The authors declare no conflict of interest.

The data that support the findings of this study are available from the corresponding author upon reasonable request. 


\section{References}

1. Cruz-Jentoft AJ, Bahat G, Bauer J, et al. European Working Group On Sarcopenia In Older People 2 (EWGSOP2), and the EXTENDED GROUP FOR EWGSOP2. Sarcopenia: revised European consensus on definition and diagnosis. Age Ageing 2019; 48: 16-31, DOI: 10.1093/ageing/afy169.

2. Doğan SC, Hizmetli S, Hayta E, et al. Sarcopenia in women with rheumatoid arthritis. Eur J Rheumatol 2015; 2: 57-61, DOI: 10.5152/eurjrheum.2015.0038.

3. Corallo C, Fioravanti A, Tenti S, et al. Sarcopenia in systemic sclerosis: the impact of nutritional, clinical, and laboratory features. Rheumatol Int 2019; 39: 1767-1777, DOI: 10.1007/ s00296-019-04401-w.

4. Korkmaz M, Eyigor S. Association between sarcopenia and rheumatological diseases. World J Rheumatol 2019; 9: 1-8, DOI: 10.5499/wjr.v9.i1.1.

5. Ngeuleu A, Allali F, Medrare L, et al. Sarcopenia in rheumatoid arthritis: prevalence, influence of disease activity and associated factors. Rheumatol Int 2017; 37: 1015-1020, DOI: 10.1007/ s00296-017-3665-x.

6. Shiboski CH, Shiboski SC, Seror R, et al. 2016 American College of Rheumatology/European League Against Rheumatism Classification Criteria for Primary Sjögren's Syndrome: a consensus and data-driven methodology involving three international patient cohorts. Arthritis Rheumatol 2017; 69: 35-45, DOI: 10.1002/art.39859.

7. Guevara-Gutiérrez E, Tlacuilo-Parra A, Minjares-Padilla LM. Minor salivary gland punch biopsy for evaluation of Sjögren's syndrome. J Clin Rheumatol 2001; 7: 401-402, DOI: 10.1097/ 00124743-200112000-00010.

8. Baumgartner R, Koehler K, Gallagher D, et al. Epidemiology of sarcopenia among the elderly in New Mexico. Am J Epidemiol 1998; 147: 755-763, DOI: 10.1093/oxfordjournals.aje.a009520 [erratum: Am J Epidemiol 1999; 149: 1161].

9. Kim KM, Jang HC, Lim S. Differences among skeletal muscle mass indices derived from height-, weight-, and body mass index-adjusted models in assessing sarcopenia. Korean J Intern Med 2016; 31: 643-650, DOI: 10.3904/kjim.2016.015.

10. Montero-Fernández N, Serra-Rexach JA. Role of exercise on sarcopenia in the elderly. Eur J Phys Rehabil Med 2013; 49: 131-143.

11. Bano G, Trevisan C, Carraro S, et al. Inflammation and sarcopenia: a systematic review and meta-analysis. Maturitas 2017; 96: 10-15, DOI: 10.1016/j.maturitas.2016.11.006.

12. Kim MK, Baek KH, Song KH, et al. Vitamin D deficiency is associated with sarcopenia in older Koreans, regardless of obesity: the fourth Korea National Health and Nutrition Examination Surveys (KNHANES IV) 2009. J Clin Endocrinol Metab 2011; 96: 3250-3256, DOI: 10.1210/jc.2011-1602.

13. Cleasby ME, Jamieson PM, Atherton PJ. Insulin resistance and sarcopenia: mechanistic links between common co-morbidities. J Endocrinol, 2016 229: R7-81, DOI: 10.1530/JOE-15-0533.

14. Kelly OJ, Gilman JC, Kim Y, llich JZ. Long-chain polyunsaturated fatty acids may mutually benefit both obesity and osteoporosis. Nutr Res 2013; 33: 521-533, DOI: 10.1016/j.nutres. 2013.04.012.

15. Argyropoulou OD, Valentini E, Ferro F, et al. One year in review 2018: Sjögren's syndrome. Clin Exp Rheumatol 2018; 36 (Suppl 112): 14-26.
16. Tagliaferri C, Wittrant Y, Davicco MJ, et al. Muscle and bone, two interconnected tissues. Ageing Res Rev 2015; 21: 55-70, DOI: 10.1016/j.arr.2015.03.002.

17. Naot D, Cornish J. Cytokines and hormones that contribute to the positive association between fat and bone. Front Endocrinol (Lausanne) 2014; 5: 70, DOI: 10.3389/fendo.2014.00070.

18. Trayhurn P, Drevon CA, Eckel J. Secreted proteins from adipose tissue and skeletal muscle - adipokines, myokines and adipose/muscle cross-talk. Arch Physiol Biochem 2011; 117: 47-56, DOI: 10.3109/13813455.2010.535835.

19. Karpe F. Insulin resistance by adiponectin deficiency: is the action in skeletal muscle. Diabetes 2013; 62: 701-702, DOI: 10.2337/db12-1519.

20. Bogl LH, Latvala A, Kaprio J, et al. An investigation into the relationship between soft tissue body composition and bone mineral density in a young adult twin sample. J Bone Miner Res 2011; 26: 79-87, DOI: 10.1002/jbmr.192.

21. Phu S, Boersma D, Duque G. Exercise and sarcopenia. J Clin Densitom 2015; 18: 488-492, DOI: 10.1016/j.jocd.2015.04.011.

22. Dufour AB, Hannan MT, Murabito JM, et al. Sarcopenia definitions considering body size and fat mass are associated with mobility limitations: the Framingham Study. J Gerontol A Biol Sci Med Sci 2013; 68: 168-174, DOI: 10.1093/gerona/gls109.

23. Baumgartner RN, Wayne SJ, Waters DL, et al. Sarcopenic obesity predicts instrumental activities of daily living disability in the elderly. Obes Res 2004; 12: 1995-2004, DOI: 10.1038/oby.2004.250.

24. Lai EL, Huang WN, Chen HH, et al. Ten-year fracture risk by FRAX and osteoporotic fractures in patients with systemic autoimmune diseases. Lupus 2019; 28: 945-953, DOI: 10.1177/ 0961203319855122.

25. Yu R, Leung J, Woo J. Sarcopenia combined with FRAX probabilities improves fracture risk prediction in older Chinese men. J Am Med Dir Assoc 2014, DOI: 10.1016/j.jamda.2014.07.011.

26. Tournadre A, Pereira B, Dutheil F, et al. Changes in body composition and metabolic profile during interleukin 6 inhibition in rheumatoid arthritis. J Cachexia Sarcopenia Muscle 2017; 8: 639-646, DOI: 10.1002/jcsm.12189.

27. Kerekes G, Nurmohamed MT, González-Gay MA, et al. Rheumatoid arthritis and metabolic syndrome. Nat Rev Rheumatol 2014; 10: 691-696, DOI: 10.1038/nrrheum.2014.121.

28. Gremese E, Tolusso B, Gigante MR, Ferraccioli G. Obesity as a risk and severity factor in rheumatic diseases (autoimmune chronic inflammatory diseases). Front Immunol 2014; 5: 576, DOI: 10.3389/fimmu.2014.00576.

29. Lazzerini PE, Capecchi PL, Bertolozzi I, et al. Marked QTc prolongation and torsades de pointes in patients with chronic inflammatory arthritis. Front Cardiovasc Med 2016; 3: 31, DOI: 10.3389/fcvm.2016.00031.

30. Samsell L, Regier M, Walton C, Cottrell L. Importance of Android/gynoid fat ratio in predicting metabolic and cardiovascular disease risk in normal weight as well as overweight and obese children. J Obes 2014; 2014: 846578, DOI: 10.1155/2014/846578.

31. Gravani F, Papadaki I, Antypa E, et al. Subclinical atherosclerosis and impaired bone health in patients with primary Sjogren's syndrome: prevalence, clinical and laboratory associations. Arthritis Res Ther 2015; 17: 99, DOI: 10.1186/s13075-015-0613-6. 